\title{
Radiation damage studies of optical link components for applications in future high energy physics experiments
}

\author{
K. Gill ${ }^{1}$, C. Aguilar ${ }^{1}$, C. Azvedo ${ }^{1}$, V. Arbet-Engels ${ }^{1}$, J. Batten ${ }^{2}$, G. Cervelli ${ }^{1}$, R. Grabit ${ }^{1}$, \\ F. Jensen ${ }^{1}$, C. Mommaert ${ }^{1}$, J. Troska ${ }^{2}$ and F. Vasey . \\ ${ }^{1}$ CERN, CH-1211, Genève 23, Switzerland. \\ ${ }^{2}$ High Energy Physics Group, Imperial College, London SW7 2BZ, UK.
}

\begin{abstract}
Optical data links are being developed at CERN for use in the tracking system of the Compact Muon Solenoid (CMS) experiment to be operated at the future CERN Large Hadron Collider. The radiation environment will be severe in the CMS tracker; simulations predict hadronic fluences $>10^{14} / \mathrm{cm}^{2}$ over an experimental lifetime of ten years, consisting of a mixture of neutrons, pions and protons over a wide energy spectrum, plus an ionizing dose of $\sim 100 \mathrm{kGy}$. Candidate optical link components must therefore be qualified for sufficient radiation hardness. Results are presented for commercially available InGaAsP lasers and InGaAs p-i-n photodiodes irradiated with $330 \mathrm{MeV}$ pions up to $5.4 \times 10^{14} \pi / \mathrm{cm}^{2}$. The evolution of the laser threshold and efficiency with fluence is presented, in addition to the leakage current and photocurrent in the photodiodes. Comparisons are drawn with previous irradiation tests on identical devices using $6 \mathrm{MeV}$ neutrons and $24 \mathrm{GeV}$ protons, and ionizing damage due to ${ }^{60} \mathrm{Co}$ gamma rays.
\end{abstract}

Keywords: Radiation damage, semiconductor lasers, p-i-n photodiodes, optical links, high energy physics.

\section{INTRODUCTION}

The Compact Muon Solenoid experiment $(\mathrm{CMS})^{1}$ is one of two large, general purpose high energy physics experiments under construction at CERN for operation in the Large Hadron Collider (LHC), which will collide two 7TeV high luminosity proton beams. Large quantities of optical fiber links are planned for analog and digital data readout as well as digital control and timing signal distribution. In the CMS tracker sub-system, analog signals from $12 \times 10^{6}$ microstrip detector channels will be time-multiplexed (at 256:1) and transmitted at 40MSamples/s along approximately 50000, 100m long, optical links. ${ }^{2,3}$ A few thousand digital optical links will also be used to transfer timing and control signals to and from the tracker.

A radiation-hard optical link featuring characteristics suitable for analog as well as digital data transmission is being developed for the CMS tracker. The link is shown schematically in Fig. 1; it is a multi-way unidirectional system based on edge-emitting laser-diode transmitters (Tx) coupled to single-mode optical fiber ribbons, multi-way MT connectors and p-i$\mathrm{n}$ photodiode receivers $(\mathrm{Rx})$. The driving and receiving electronics is designed according to the system application: analog for the readout-, and digital for the control-system of the CMS tracker.

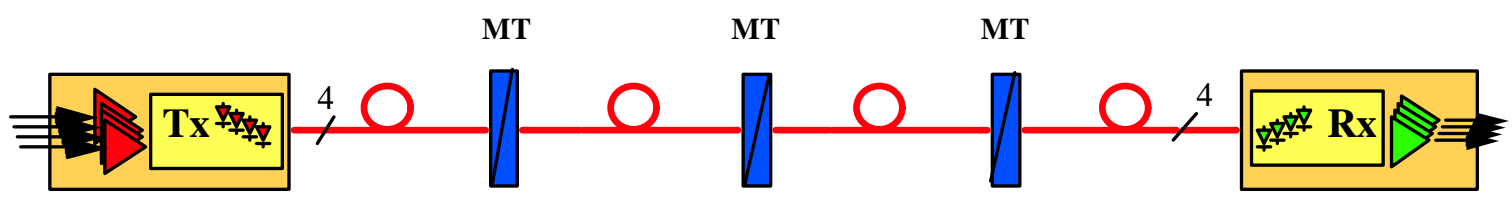

Fig. 1: Generic optical link block diagram.

The optoelectronic transmitter and receiver components are assembled using Si-submount technology. The laser diodes are commercially available Multi-Quantum-Well (MQW) InGaAsP edge-emitting devices selected for their good linearity, low 
threshold current $(\sim 9 \mathrm{~mA})$ and proven reliability. Photodiodes are epitaxially grown, planar InGaAs devices of small active volume. All the measurements reported below have been performed using components housed in one-way mini-Dual-InLine (mini-DIL) packages, but the final system will be based on assemblies of up to four laser- or pin-diode submounts housed in a single custom developed package approximately $15 \mathrm{~mm} \times 10 \mathrm{~mm} \times 4 \mathrm{~mm}$ in volume.

The LHC beams (actually intense bunches of protons) will be collided at a rate of 40MHz. At the highest beam luminosity $\left(\sim 10^{34} / \mathrm{cm}^{2} / \mathrm{s}\right)$ there will be, on average, $20 \mathrm{p}$-p collisions in each bunch crossing, producing $\sim 3000$ high energy particles. The majority of these particles travel down the beam pipe without hitting any sensitive material, but those emitted with sufficient transverse momentum are capable of causing significant radiation damage to the detectors and associated materials inside the experiment. The particle fluences inside CMS will be highest close to the beam interaction point, in the tracking detector volume. For example, at a radius of $20 \mathrm{~cm}$ from the beam axis, the fluence over ten years of high luminosity operation will consist of $\sim 10^{14}$ neutrons $/ \mathrm{cm}^{2}(\sim 1 \mathrm{MeV})$ and $\sim 2 \times 10^{14} / \mathrm{cm}^{2}$ charged hadrons $(75 \%$ pions, $15 \%$ protons and $15 \%$ kaons) with energies in the range of $100 \mathrm{MeV}$ to $10 \mathrm{GeV} .^{2}$ In addition, a total ionizing dose of $\sim 100 \mathrm{kGy}$ will result from energy deposited by charged particles and gammas.

For the analog optical links, lasers, fibers and connectors (as well as front-end electronics) will be positioned in the tracker and exposed to radiation, whereas the receiver (back-end) will not be exposed to any radiation. For the digital links, which transmit data to and from the tracker, both lasers and photodiodes (and associated electronics) will be located in the radiation zone. Validation of candidate link components to LHC fluence and dose levels is therefore an important part of the optical link development and we have measured the effects of particle fluences and doses typical of 10 years of high luminosity running for a variety of radiation sources. Clearly, experiments with low dose rate to high doses are impractical and we have therefore had to carry out accelerated irradiation tests, over more convenient periods of 10-100 hours, in addition to measuring the annealing characteristics after irradiation. With both damage and annealing data it is then possible to extrapolate to a longer irradiation duration.

In this article we summarize the latest irradiation test using $330 \mathrm{MeV}$ pions. ${ }^{5,6}$ These particles will dominate the radiation flux of hadronic particles in the tracker volume closest to the beam collision point. The energy of $330 \mathrm{MeV}$ is at the peak of the LHC pion emission spectrum ${ }^{2}$ and it is also at a resonance in the pion-nucleon interaction cross-section ${ }^{4}$ where enhancements in the displacement damage rate may occur. In addition to pion irradiation, we have also tested identical lasers and p-i-n photodiodes using other radiation sources that are similar to the different elements of the particle spectra expected inside the CMS tracker. A ${ }^{60} \mathrm{Co}$ gamma source (doses to $100 \mathrm{kGy}$ ) was used to measure the effect of the ionizing radiation encountered within the tracker. A $6 \mathrm{MeV}$ (mean-energy) neutron source has been used to measure the damage from neutrons with energies typical of spallation reactions (the source of the $\sim 1 \mathrm{MeV}$ neutron flux throughout much of the tracker), and a $24 \mathrm{GeV}$ proton beam has been used to determine whether the high energy end of the LHC hadron spectrum causes a greater amount of damage per incident particle. We will concentrate on the pion damage measurements and a comparison will be made of the damage effects between pions and the other radiation types investigated.

In semiconductor lasers and p-i-n diodes, we have found that the effects of displacement damage are much more important than ionization damage for the fluences and doses typical of LHC levels. ${ }^{7}$ This type of damage occurs when an incident particle transfers sufficient energy in a collision with a host atom to displace it from its lattice position. Given sufficient energy transfer, the recoiling atom can then produce many further atomic displacements as it comes to rest. Displacement damage introduces defect states into the semiconductor band-gap that can act as generation-recombination centers. In lasers, recombination at these defects competes with band-to-band radiative transitions, resulting in higher threshold currents. ${ }^{7-9}$ The slope-efficiency above threshold $(\mathrm{dP} / \mathrm{dI}$, where $\mathrm{P}$ is the output light power at current $\mathrm{I})$ is less affected by displacement damage as the stimulated recombination lifetime is much shorter than the lifetime associated with recombination at defects. However the damage to the laser slope-efficiency becomes significant after high hadron fluences $\left(>10^{14} / \mathrm{cm}^{2}\right)$ and has been linked directly to the threshold current damage. ${ }^{7}$ It is therefore likely that the same type of defect is responsible for both of these damage effects in lasers. In p-i-n photodiodes, generation of charge at the defects causes an increase in dark current, which raises the minimum power level of detectable optical signals and increases the noise in the optical link. A defect at $\mathrm{E}_{\mathrm{c}}-0.29 \mathrm{eV}$ in the band-gap is reported to be responsible for leakage current increases in electron irradiated InGaAs p-i-n diodes ${ }^{10}$ and it is likely that the same defect is present after neutron (and presumably other heavy particle) damage. ${ }^{11}$ Radiation induced defect states in the band-gap also act as trapping/recombination centers for photoinduced signal charge, causing a reduction of the photocurrent. ${ }^{7,11,12}$ All of the damage effects described above were strongly evident after pion irradiation to the fluence levels expected in the CMS tracker. 


\section{EXPERIMENT}

Five lasers and five p-i-n photodiodes were irradiated with pions $\left(\pi^{+}\right)$at the Paul Scherrer Institute (PSI), Villigen, Switzerland. The lasers were NEC 1310nm low-threshold edge-emitting InGaAsP multi-quantum-well (MQW) doublechannel planar heterostructure (DCPBH) devices, housed in a mini-DIL package supplied by Italtel, pigtailed with singlemode (SM) fiber, terminated with a standard FC/PC connector. Pre-irradiation values of the threshold current were $~ 9 \mathrm{~mA}$, with output efficiency of $60 \mu \mathrm{W} / \mathrm{mA}$. The p-i-n photodiodes were Epitaxx back-illuminated $75 \mu \mathrm{m}$ diameter InGaAs/InP diodes mounted in identical mini-DIL packages to the lasers, also pigtailed with SM fiber terminated with a FC/PC connector. The pre-irradiation levels of the leakage current (at $-5 \mathrm{~V}$ ) were $<0.01 \mathrm{nA}$ and the responsivity was $\sim 0.9 \mathrm{~A} / \mathrm{W}$. The packaged devices used in these tests did not include integrated sub-elements, such as lenses, that can be degraded by radiation damage. ${ }^{13}$

The devices were arranged in a stack aligned along the beam such that the device active layers were perpendicular to the pion beam. The beam was focused on the front of the stack and the pion flux was approximately constant at $1-2 \times 10^{9} \pi / \mathrm{cm}^{2} / \mathrm{s}$ depending upon the exact position. The total fluence received by the devices over an irradiation period of 96 hours was between $2.8 \times 10^{14} \pi / \mathrm{cm}^{2}$ and $5.4 \times 10^{14} \pi / \mathrm{cm}^{2}$ Dosimetry was done with $5 \times 5 \mathrm{~mm}^{2}$ aluminum foils, ${ }^{5}$ placed at several positions in the stack between the lasers and p-i-n photodiodes. The fluence for each device was then determined by interpolation with a precision of $10 \%$. The irradiation was carried out at ambient temperature and values of $18-20^{\circ} \mathrm{C}$ were measured in the beam area during irradiation. Following exposure, the devices were taken out of the beam and measured at 1 hour intervals for one month (under the same bias conditions as for irradiation, with no modifications to the optical connections). Further measurements of the damage annealing were later continued at CERN using higher temperatures between 40 and $80^{\circ} \mathrm{C}$ to accelerate the recovery process. ${ }^{5,6}$

During the pion irradiation the laser L-I (output light power versus current) characteristics were monitored in-situ at intervals of 30 minutes. The laser threshold current and slope efficiency were then determined from the L-I characteristics. Three of the five lasers irradiated with pions were biased throughout the test at 5-10mA above threshold and the other two devices were unbiased (shorted to ground), except during measurement of the device characteristics. For the five p-i-n photodiodes, the leakage current and photocurrent were also measured at 30 minute intervals, at five different reverse bias voltages: $0 \mathrm{~V}, 2.5 \mathrm{~V}, 5 \mathrm{~V}, 7.5 \mathrm{~V}$ and $10 \mathrm{~V}$. The photodiodes were illuminated using a $1310 \mathrm{~nm}$ laser situated outside the beam area in a stable temperature environment. The photocurrent was measured for optical power levels between 0 and $500 \mu \mathrm{W}$ by monitoring the $\mathrm{p}-\mathrm{i}-\mathrm{n}$ current whilst ramping the current through the external laser. An external p-i-n diode was used to monitor any fluctuations in the laser power output.

\section{DAMAGE RESULTS}

\subsection{Lasers}

The damage due to pion irradiation to the laser L-I characteristics is illustrated in Fig. 2 (for laser 1) at different times during the irradiation. The increase in threshold current and loss of efficiency, consistent with a decrease in injected carrier lifetimes, are both clearly visible. The threshold current increase and efficiency loss are shown for all five devices as a function of pion fluence (without any corrections for annealing during the exposure) in Figs. 3 and 4. The overall threshold shifts and efficiency losses are larger than those observed in $6 \mathrm{MeV}$ neutron irradiation and similar to those obtained in $24 \mathrm{GeV}$ proton irradiation. A more detailed comparison is given in Section 4. 


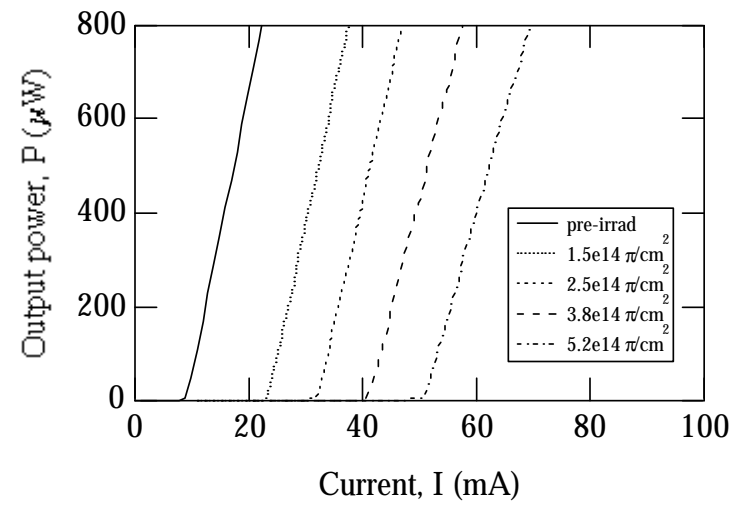

Fig. 2: Change in L-I characteristics in laser 1 due to pion irradiation. Data points were measured at $1 \mathrm{~mA}$ intervals.

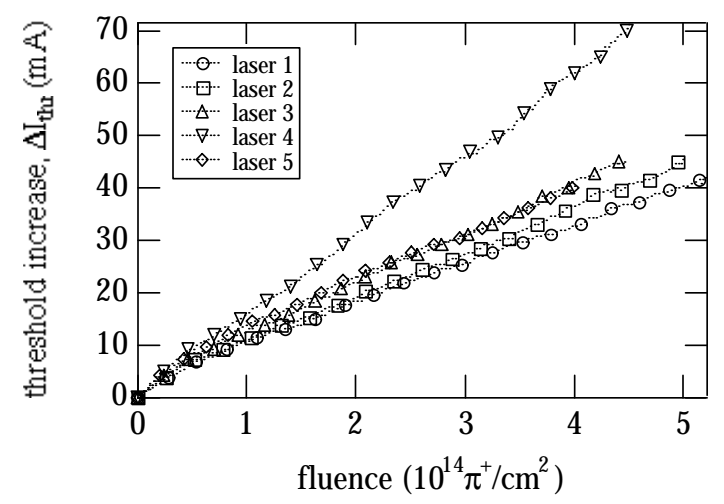

Fig. 3: Laser threshold increase versus pion fluence.

Pre-irradiation threshold current values were typically $9 \mathrm{~mA}$.

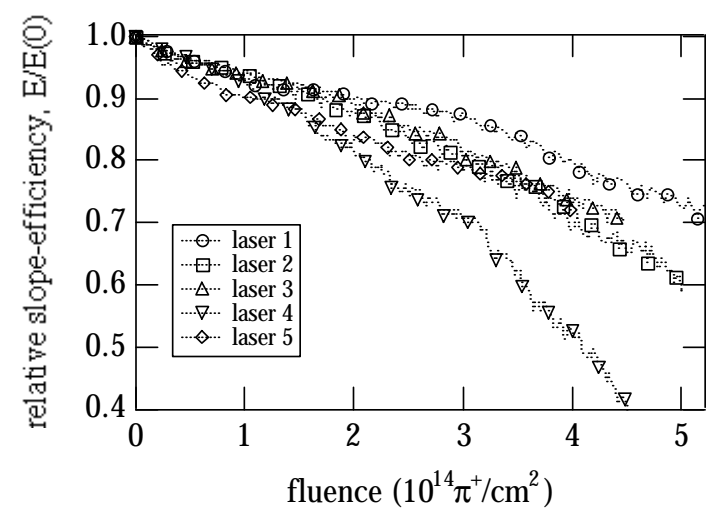

Fig. 4: Slope-efficiency decrease versus pion fluence. Data normalized to pre-irradiation values which were typically $60 \mu \mathrm{W} / \mathrm{mA}$. 
The data for the biased lasers (1-3) are all in good agreement, whereas there is a large difference in the damage effects in the two unbiased devices ( 4 and 5); laser 4 has almost twice the damage of laser 5 (and lasers 1-3). This difference is outside the range of dosimetry errors and other known experimental uncertainties. In the earlier neutron and proton irradiation studies the effect of biasing the devices consistently reduced the damage by 25-30\% (see Section 4), so it would appear as though laser 4 is more damaged and laser 5 is less damaged than expected. Electrically biasing the devices generally decreases the degree of radiation damage by increasing the amount of annealing that takes place during and after irradiation. This effect is known as 'injection-enhanced annealing', as observed in other irradiation studies on lasers ${ }^{14}$ and LEDs ${ }^{15}$ with the magnitude of the enhancement varying depending upon the particular type of device and semiconductor material.

The annealing of the threshold current at room temperature was monitored for 610 hours following irradiation and 30-40\% of the total damage annealed during this time. Further measurements of the annealing were then made at temperatures of $40^{\circ} \mathrm{C}, 60^{\circ} \mathrm{C}$ and $80^{\circ} \mathrm{C}$ (with 260 hours at each step). The overall effect of this accelerated annealing was to recover a further $25-35 \%$ of the damage. No anti-annealing was observed (where the damage effects continue to increase in the period following irradiation), as observed in other irradiated semiconductor devices such as high resistivity silicon diodes. ${ }^{16}$ Another experiment is planned to measure the annealing more carefully, over a range of temperatures, in order to determine the activation energies associated with the annealing.

In terms of the operation of lasers in the final CMS optical link system, the radiation damage to the lasers appears to be tolerable. The linearity of the device L-I characteristic remains good enough for analog transmission even after high fluences. The threshold increases can be compensated by the laser driver circuit, which is specified ${ }^{17}$ to track changes in the threshold up to 50-60mA. This range should be sufficient to compensate for the expected threshold increases, since the pion fluences used in this test are several times higher than those expected inside the CMS tracker and annealing effects will suppress the damage over an irradiation period of $\sim 10$ years. The radiation induced efficiency loss is also acceptable in terms of the CMS analog link application as gain fluctuations will be compensated by periodic recalibration of the electronic readout chain.

\subsection{P-i-n photodiodes}

The effect of pion damage on the p-i-n photodiode characteristics is illustrated in Fig. 5 (for p-i-n number 1), showing a large increase in leakage current and decrease in responsivity for various pion fluences during the test. The effects are qualitatively similar to the damage measured in earlier neutron and proton tests, with the rate of pion damage being similar to $24 \mathrm{GeV}$ protons and several times greater than $6 \mathrm{MeV}$ neutrons as detailed in Section 4 .

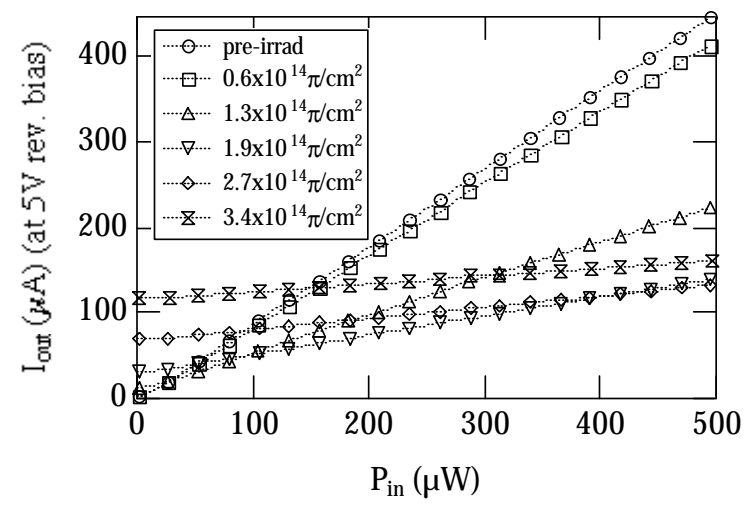

Fig. 5: Degradation of the p-i-n characteristics due to $330 \mathrm{MeV}$ pion damage.

The increase in the leakage current with fluence (the fluence was linear with time during the irradiation) is shown, along with the decrease in photocurrent (for $100 \mu \mathrm{W}$ optical signals) for various p-i-n reverse bias voltages in Figs. 6 and 7 respectively. In addition to these damage data, similar measurements were made after irradiation to map the annealing effects. About $15 \%$ of the leakage current damage annealed in 610 hours after irradiation at room temperature. No significant recovery of the responsivity damage was observed during the same period. In subsequent higher temperature 
annealing steps $\left(260\right.$ hours at $40^{\circ} \mathrm{C}, 60^{\circ} \mathrm{C}$ and $80^{\circ} \mathrm{C}$ as for the lasers) a further $20 \%$ of the leakage current damage was recovered but $<1 \%$ of the photocurrent damage annealed.

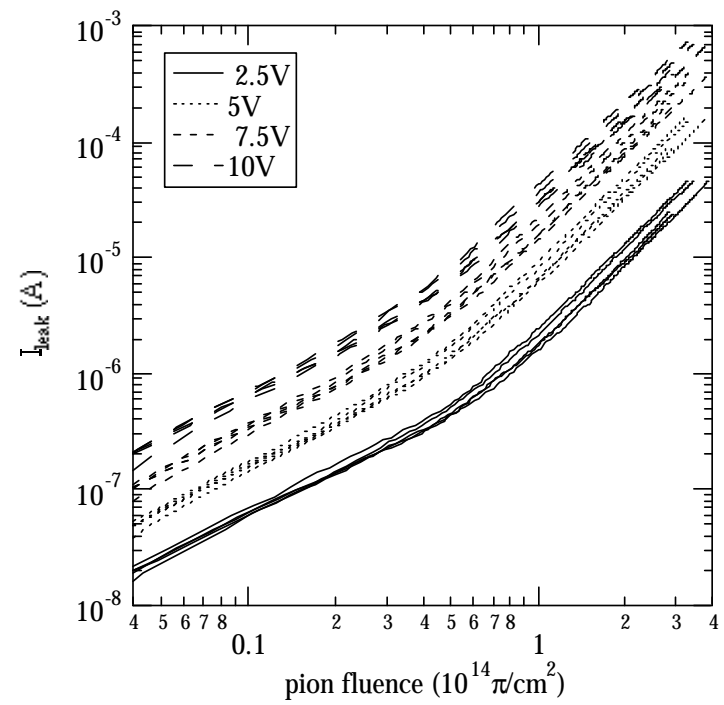

Fig. 6: Leakage current increase in p-i-n photodiodes due to pion damage.

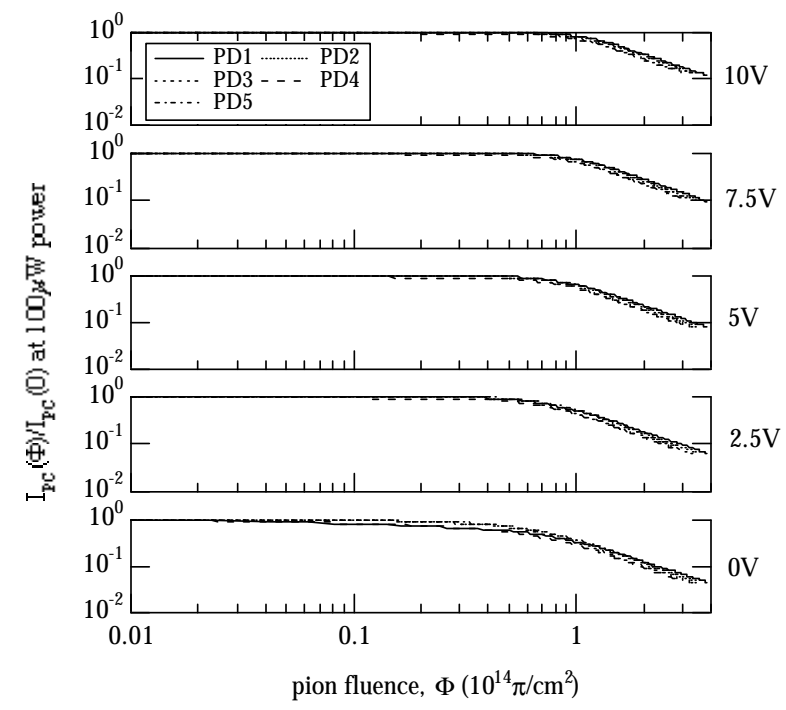

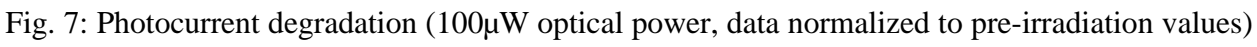
in pion irradiated $\mathrm{p}-\mathrm{i}-\mathrm{n}$ photodiodes.

The damage effects in the p-i-n photodiodes are consistent with the build-up of radiation induced defects in the bulk leading to the generation of dark current and the trapping/recombination of signal charge. It should be emphasized that the sharp decrease in photocurrent response that occurs after $\sim 5 \times 10^{13} \pi / \mathrm{cm}^{2}$ is particular to these devices. In our recent neutron damage studies, using both front-illuminated and back-illuminated Epitaxx InGaAs p-i-n photodiodes of identical geometry, the responsivity of the front-illuminated devices remained high ( $80 \%$ of the initial value) up to the tested fluence of $10^{15} \mathrm{n} / \mathrm{cm}^{2}$ whereas a sharp decrease in the photocurrent was measured in the back-illuminated devices after $3 \times 10^{14} \mathrm{n} / \mathrm{cm}^{2}$. The reason for this difference in the radiation hardness between front- and back-illuminated devices is currently being investigated, but the effects may be due, for example, to a build-up of predominantly acceptor-type defects in the bulk. This would eventually lead to 'type-inversion' of the nominally intrinsic (but actually lightly-doped n-type) InGaAs layer from n-type to p-type. Type-inversion of the InGaAs layer after $\sim 5 \times 10^{13} \pi / \mathrm{cm}^{2}$ would explain the sharp change in the response at this fluence, as uncompensated negatively charged acceptors in the InGaAs layer would be 
effective trapping centers for signal induced holes after type-inversion. Due to the short optical absorption length of InGaAs at $1.3 \mu \mathrm{m}$, this would be more damaging in back-illuminated p-i-n diodes than in front-illuminated devices because signal induced holes must travel a greater distance through the InGaAs layer in back-illuminated diodes.

In terms of the digital link operation inside the CMS tracker, the radiation induced leakage currents may approach the amplitude of the optically-induced signals in the digital links $(\sim 100 \mu \mathrm{A}$ before radiation-induced attenuation). The link receiver electronics therefore have to compensate for leakage currents up to $\sim 100 \mu \mathrm{A}$. For the responsivity loss, even though the damage effect is apparently very large for these devices, we have demonstrated a bit-error-rate $<10^{-12}$ in a $40 \mathrm{Mb} / \mathrm{s}$ digital link using a neutron damaged $\mathrm{p}-\mathrm{i}-\mathrm{n}$ diode of the same type (with $70 \%$ responsivity loss). ${ }^{3}$

\section{COMPARISON OF DAMAGE IN PION, NEUTRON AND PROTON TESTS}

\subsection{Lasers}

Devices of the same type have now been irradiated with pion, proton, neutron and gamma sources. Gamma damage will not be discussed further as the effects were not significant compared to hadron damage, for dose/fluence levels expected during LHC operation. The data from pion irradiation can be compared directly with that from neutron irradiation since the duration of the irradiation tests were the same ( $\sim 96$ hours). For the comparison with the proton irradiation, the proton damage data have been calculated based on an extrapolation of the actual data ${ }^{7}$ (obtained in a 10.5 hours exposure to $4 \times 10^{14} \mathrm{p} / \mathrm{cm}^{2}$ ) to a fluence of $5 \times 10^{14} \mathrm{p} / \mathrm{cm}^{2}$ in an exposure time of 96 hours. This takes into account the extra annealing during an irradiation over 96 hours. For pion, neutron and proton irradiation we have assumed that the same type of defect species were introduced, in the same relative proportions, and that the introduction of defects is linear with fluence as the data suggests.

Fig. 8 compares the threshold damage from pions to that resulting from $6 \mathrm{MeV}$ neutron and $24 \mathrm{GeV}$ proton irradiation. The neutron data is for eight biased lasers plus two unbiased devices irradiated for 96 hours. The proton test used five lasers, three biased and two shorted during irradiation. In all the irradiation tests, the biased devices were operated typically 5$10 \mathrm{~mA}$ above threshold during the exposure. Table 1 shows the relative damage factors for pions, neutrons and protons.
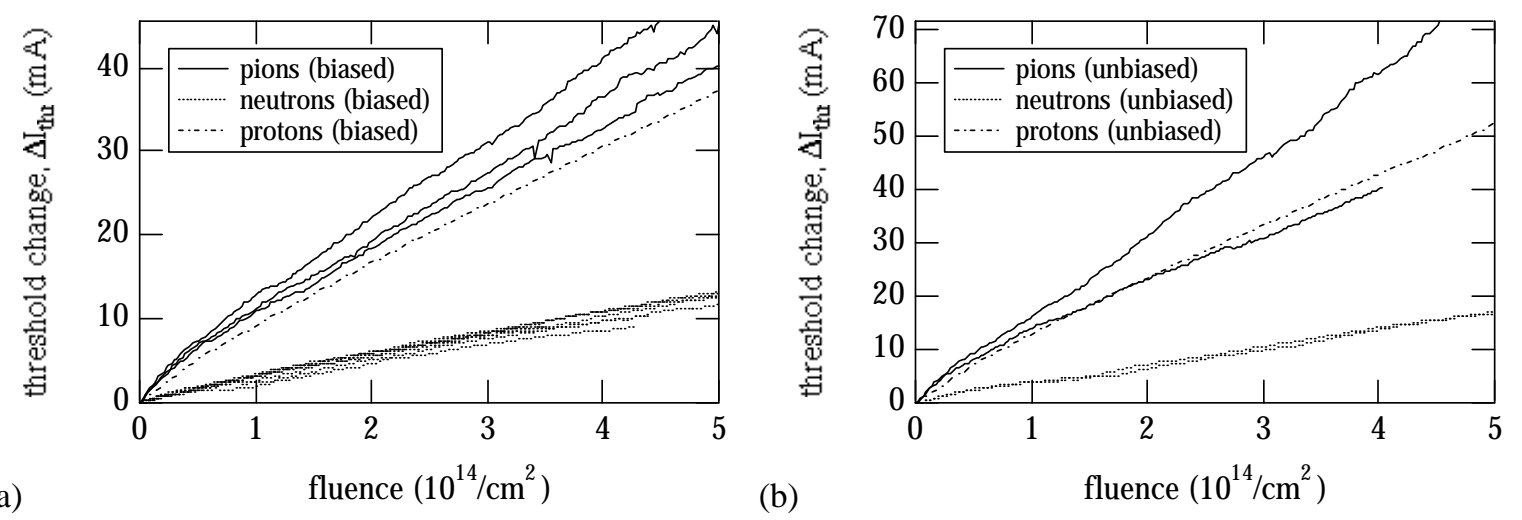

Fig. 8: Threshold increase comparison between $330 \mathrm{MeV}$ pion, $6 \mathrm{MeV}$ neutron and $24 \mathrm{GeV}$ proton irradiation in InGaAsP lasers (a) biased 5-10mA above threshold during irradiation and (b) shorted during irradiation.

\begin{tabular}{|c|c|c|}
\cline { 2 - 3 } \multicolumn{1}{c|}{} & $\pi / \mathrm{n}$ factor & $\pi / \mathrm{p}$ factor \\
\hline biased & $3.9( \pm 20 \%)$ & $1.2( \pm 15 \%)$ \\
\hline unbiased & $3.7( \pm 30 \%)$ & $1.2( \pm 20 \%)$ \\
\hline
\end{tabular}

Table 1: Relative damage factors in InGaAsP lasers for $330 \mathrm{MeV}$ pions, $6 \mathrm{MeV}$ neutrons and $24 \mathrm{GeV}$ protons. 
In both pion and proton irradiation tests, the devices were oriented with the active layer perpendicular to the incident beam, whereas in the neutron tests the devices were placed such that the active layer was parallel to the beam. For $6 \mathrm{MeV}$ neutron damage no significant dependence on incident particle angle has been observed. However, recent measurements on GaAs lasers with higher energy particles $(300 \mathrm{MeV}$ protons) indicate a $50 \%$ increase in damage when the incident radiation direction is parallel to the active layers rather than perpendicular. ${ }^{18}$ We plan to carry out a test to determine the angular dependence of damage due to high energy hadrons in our lasers, as optical link components inside the CMS tracker will be positioned at various angles relative to the incident particle flux.

\subsection{P-i-n photodiodes}

As with the lasers, p-i-n diodes of the same type have now been irradiated under similar conditions (-5V bias at room temperature) with pion, neutron, proton and gamma sources. The overall effects of leakage current increase and responsivity loss are qualitatively similar for pions, neutrons and protons. Damage resulting from ${ }^{60} \mathrm{Co}$ gamma irradiation was negligible in comparison, with $\sim 10 \mathrm{nA}$ leakage current (at $-5 \mathrm{~V}$ bias) and no signal loss after $100 \mathrm{kGy},{ }^{11}$ so gamma damage will not be discussed further. As for the laser comparison above, the pion and neutron data can be compared directly as the exposures were of the same duration, 96 hours. The proton irradiation was over only 10.5 hours and the proton damage data should be corrected for annealing to compare with results obtained in an exposure of 96 hours. However, due to the non-linear dependence of damage on fluence, it is not possible to extrapolate precisely the proton damage to a longer exposure time and a direct comparison has to be made for a given fluence. Since only $20 \%$ of the proton induced leakage current damage annealed in 100 hours after the $10.5 \mathrm{hr}$ irradiation, ${ }^{7}$ the systematic error in the pion to proton comparison will remain relatively small $(\sim 10 \%$ over-estimate of the proton damage). For the photocurrent measurements, no corrections are necessary for the different exposure times since no significant annealing of the responsivity damage occurred at room temperature following the pion, proton and neutron irradiation tests (even over periods of several months).

The damage resulting from $330 \mathrm{MeV}$ pion irradiation is compared to that from $6 \mathrm{MeV}$ neutrons and $24 \mathrm{GeV}$ protons in Figs. 9 and 10 for the leakage current and photocurrent respectively. Five devices were irradiated with neutrons and four with protons, all under $5 \mathrm{~V}$ reverse bias and illumination conditions identical to the pion test. Due to the non-linear build-up of damage, any comparisons have to be made in terms of the fluence required to produce a certain level of damage, as opposed to the more direct comparison of damage increase per unit fluence. The damage due to $330 \mathrm{MeV}$ pions is found to be very similar to that from $24 \mathrm{GeV}$ protons, for both leakage and photocurrent damage, and about 10 times greater than $6 \mathrm{MeV}$ neutrons in terms of leakage current and around 4-5 times greater in terms of photocurrent damage.

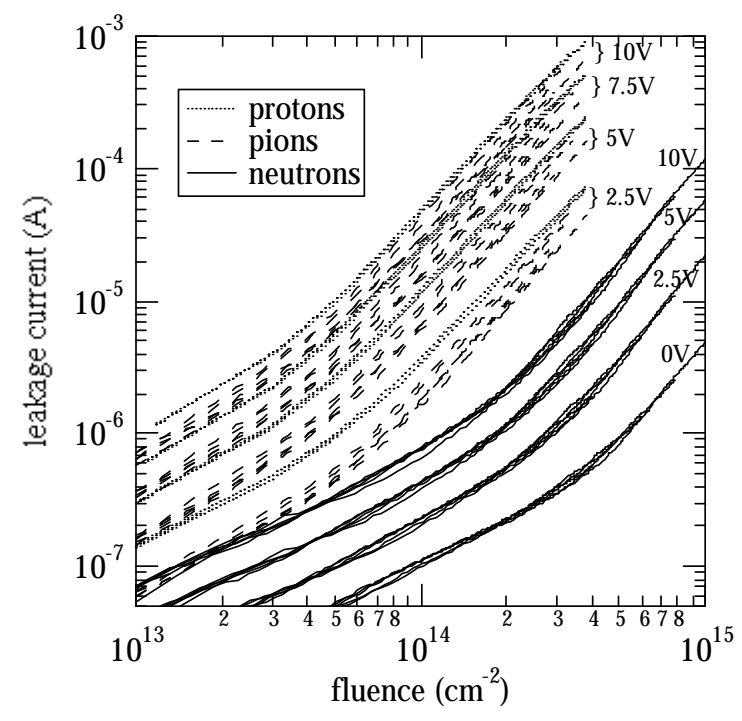


Fig. 9: Comparison of leakage current damage in p-i-n photodiodes due to $330 \mathrm{MeV}$ pion, $6 \mathrm{MeV}$ neutron and $24 \mathrm{GeV}$ proton irradiation. Pre-irradiation values were $\sim 10 \mathrm{pA}$ at $-5 \mathrm{~V}$.

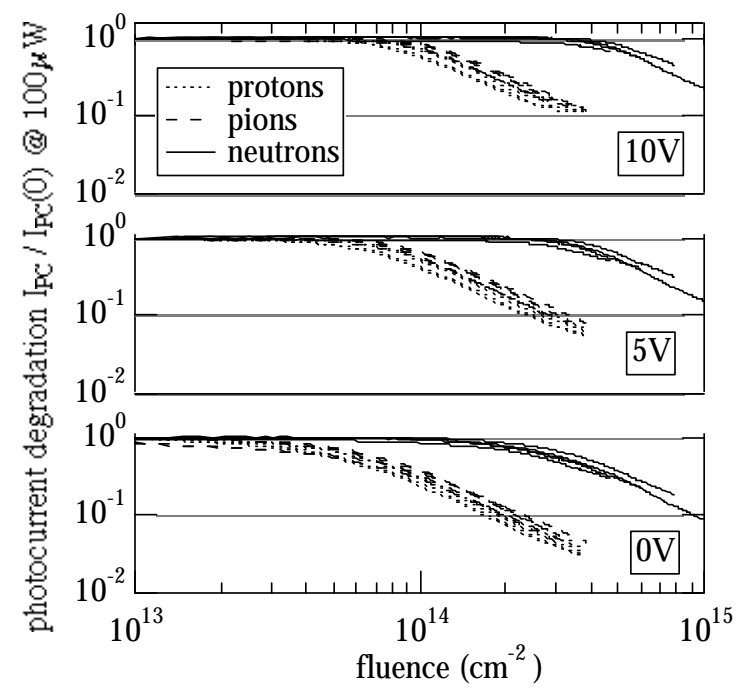

Fig. 10: Comparison of photocurrent degradation in p-i-n photodiodes irradiated with $330 \mathrm{MeV}$ pions, $6 \mathrm{MeV}$ neutrons and $24 \mathrm{GeV}$ protons. The photocurrent is shown for $100 \mu \mathrm{W}$ optical power, with the data normalized to the pre-irradiation signal level ( $90 \mu \mathrm{A})$.

\subsection{Non-ionizing energy loss of recoiling atoms}

It is interesting to note that the large difference observed between the effects of $6 \mathrm{MeV}$ neutrons and those due to $24 \mathrm{GeV}$ proton and $330 \mathrm{MeV} / \mathrm{c}$ pions is qualitatively similar to that observed in irradiated GaAs detectors, where the ratio is 1:3.1:3.8 for $1 \mathrm{MeV}$ neutrons, $24 \mathrm{GeV}$ protons and $330 \mathrm{MeV}$ pions respectively. ${ }^{19}$ In contrast, the damage ratios are all very similar in silicon detectors irradiated with the same three radiation sources (n:p: $\pi=1: 0.93: 0.93){ }^{20}$ The explanation for this difference in damage ratios between $\mathrm{Si}$ and GaAs is related to the masses of the constituent elements and the fraction of the kinetic energy of recoiling atoms/nuclei that is dissipated in further atomic displacement, i.e. the non-ionizing energy loss (NIEL). ${ }^{21}$ The differences we observe for the damage to lasers and $\mathrm{p}-\mathrm{i}-\mathrm{n}$ photodiodes between pion, proton and neutron damage are also expected to be due to different NIEL values.

The NIEL fraction of the recoil energy is larger for heavier atoms for a given recoil energy. This is illustrated in Fig. 11 which shows the amount of recoil energy that is non-ionizing in nature, using the Lindhard theory for stopping power 22,23 for In, Ga, As, and P recoils in InGaAsP compared to Si recoils in silicon bulk material. The InGaAsP stoichiometry is assumed for this calculation to be $\mathrm{In}_{0.71} \mathrm{Ga}_{0.29} \mathrm{As}_{0.61} \mathrm{P}_{0.39}$ and small deviations in the composition should not significantly affect these results. The maximum NIEL of a recoiling Si atom in Si bulk material in the energy range shown is found to be $\sim 200 \mathrm{keV}$, compared to a maximum value of $\sim 2 \mathrm{MeV}$ for In in InGaAsP. $1 \mathrm{MeV}$ neutrons can provide a recoil energy up to $130 \mathrm{keV}$ in silicon in an elastic collision, of which $100 \mathrm{keV}$ is deposited as NIEL. This is already close to the limit of maximum NIEL per recoil in silicon and higher energy incident particles are therefore not expected to cause much more damage in silicon.

In the case of InGaAsP, the maximum recoil energy from $1 \mathrm{MeV}$ neutrons of a $\mathrm{Ga}$ atom for instance is $\sim 60 \mathrm{keV}$, typically dissipating $40 \mathrm{keV}$ of this energy in further atomic displacements. Since the NIEL of recoils in InGaAsP can be up to several $\mathrm{MeV}$, it is clear that higher energy protons and pions will generate more damage per recoiling atom than a $1 \mathrm{MeV}$ neutron, in contrast to the situation in silicon devices. The same NIEL arguments should also apply to the InGaAs photodiodes as for the lasers, because the devices are of similar material, and it is therefore expected that the higher energy pions and protons will cause several times more damage than the $6 \mathrm{MeV}$ neutrons, as observed. More detailed quantitative calculations of NIEL are necessary that should also include the laser (or p-i-n) diode geometry, the different interaction cross-sections, and the full spectra of recoil energies. This would allow us confirm whether the observed damage in lasers and $\mathrm{p}-\mathrm{i}-\mathrm{n}$ photodiodes is proportional to NIEL, and to make predictions for the radiation damage expected during CMS operation. 


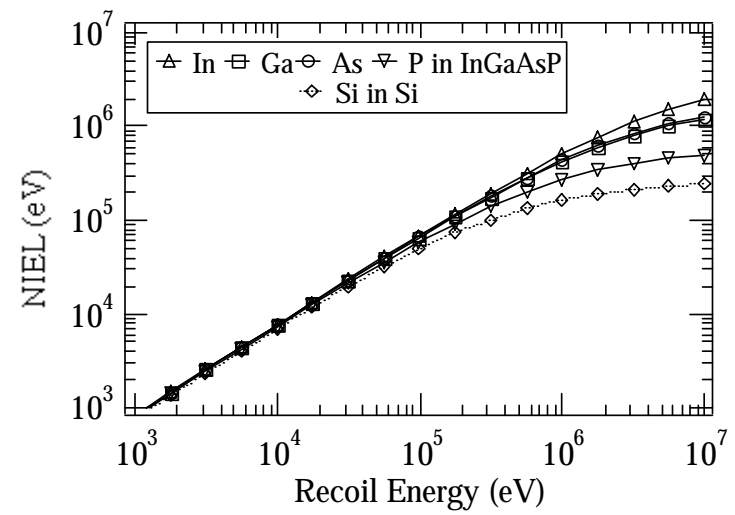

Fig. 11: Non-ionizing energy loss of recoiling atoms in different bulk materials, as a function of the recoil energy of the atom for In, Ga, As and P atoms recoiling in InGaAsP laser material, compared to $\mathrm{Si}$ recoils in bulk silicon.

\section{CONCLUSION}

In the innermost parts of the CMS tracker the radiation flux will be dominated by pions with $\sim 300 \mathrm{MeV}$ energy. We have measured the radiation damage effects due to $330 \mathrm{MeV}$ pions $\left(\pi^{+}\right)$on lasers and $\mathrm{p}-\mathrm{i}-\mathrm{n}$ photodiodes for use in the tracker optical readout and digital control/timing links. Five lasers and five photodiodes mounted in mini-DIL packages were irradiated at room temperature with pion fluences between 2.8 and $5.4 \times 10^{14} \pi / \mathrm{cm}^{2}$. The pion damage effects are summarized in Table 2 for a fluence of $2 \times 10^{14} \pi / \mathrm{cm}^{2}$, which is typical of 10 years of operation at $20 \mathrm{~cm}$ radius from the LHC beam axis. This essentially represents a worst-case as most of the links will be located at greater radial distances from the beam interaction point and will therefore be exposed to much lower particle fluences. In addition, the build-up of damage will also be suppressed due to greater annealing over a 10 year irradiation period. Further tests are planned to accurately determine the annealing rates for both lasers and p-i-n diodes as a function of temperature, as the innermost (silicon) part of the CMS tracker will be maintained at $-10^{\circ} \mathrm{C}$ and the outer layers of (gas microstrip) tracking detectors will be operated at $20^{\circ} \mathrm{C}$.

\begin{tabular}{|c|c|c|}
\hline & pre-irradiation & after $2 \times 10^{14} \pi / \mathrm{cm}^{2}$ \\
\hline laser threshold current & $9 \mathrm{~mA}$ & $\begin{array}{c}19 \mathrm{~mA} \text { (biased) } \\
26 \mathrm{~mA} \text { (unbiased) }\end{array}$ \\
\hline laser slope-efficiency & $60 \mu \mathrm{W} / \mathrm{mA}$ & $\begin{array}{c}54 \mu \mathrm{W} / \mathrm{mA} \text { (biased) } \\
51 \mu \mathrm{W} / \mathrm{mA} \text { (unbiased) }\end{array}$ \\
\hline p-i-n leakage (@ 5V) & $<0.5 \mathrm{nA}$ & $40 \mu \mathrm{A}$ \\
\hline 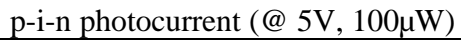 & $90 \mu \mathrm{A}$ & $18 \mu \mathrm{A}$ \\
\hline
\end{tabular}

Table 2: Summary of average amounts of $330 \mathrm{MeV}$ pion damage after $2 \times 10^{14} \pi / \mathrm{cm}^{2}$ in InGaAsP lasers and InGaAs p-i-n photodiodes

The damage effects in both lasers and photodiodes are most likely due to displacement damage in the active region leading to enhanced generation-recombination at defect states in the band-gap. In both lasers and p-i-n photodiodes there is more damage from the pions (and protons) relative to the neutrons, which is in contrast to the similar relative damage levels observed in irradiated silicon devices. In terms of laser threshold current damage, $330 \mathrm{MeV}$ pions are 1.2 times more damaging than $24 \mathrm{GeV}$ protons and 3.8 times worse than $6 \mathrm{MeV}$ neutrons. For the p-i-n photodiodes, $330 \mathrm{MeV}$ pions caused a similar amount of damage as $24 \mathrm{GeV}$ protons and were several times more damaging then $6 \mathrm{MeV}$ neutrons, with the precise factor dependent upon the amount of damage being compared. The large difference in the damage factor between $330 \mathrm{MeV}$ pions (and $24 \mathrm{GeV}$ protons) relative to $6 \mathrm{MeV}$ neutrons is consistent with NIEL arguments and a more detailed model is being developed to calculate the NIEL in the lasers and p-i-n diodes. In addition, a further high energy proton test is planned to measure the effect of incident particle direction on the radiation damage. 
In terms of operating the analog optical readout links in the final system, the laser threshold shifts can be tolerated as the laser driver is capable of tracking increases of $50-60 \mathrm{~mA}$ under the current specifications. The radiation induced loss of slope-efficiency in the lasers is not sufficient to cause concern and the final system will be periodically recalibrated to correct for any fluctuations in the gain of the readout chain. For the digital control and timing distribution links, where p-i$\mathrm{n}$ diodes will be situated in the tracker volume, the increases in leakage current will be compensated by the receiver electronics, and the loss of response will not significantly affect the bit-error-rate at $40 \mathrm{Mb} / \mathrm{s}$. The photocurrent degradation in the p-i-n photodiodes is specific to this type of back-illuminated device and, in our other recent tests, front-illuminated photodiodes have been found to be more radiation-hard.

\section{ACKNOWLEDGMENTS}

We wish to thank Bernard Cornet and Loic Baumard for preparing the test hardware; Francois Lemeilleur, Maurice Glaser and Mika Huhtinen of CERN for very useful advice; Kurt Gabathuler of PSI for arranging the pion irradiation, and the Vienna and Dortmund groups for their assistance during the pion test.

\section{REFERENCES}

1. The Compact Muon Solenoid Technical Proposal. CERN/LHCC 94-38, 1994.

2. CMS Tracker Technical Design Report, CERN/LHCC 98-6, CMS TDR 5, 1998.

3. F. Vasey et al, "Development of radiation-hard optical links for the CMS tracker at CERN", Proceedings of IEEE Nuclear Science Symposium, 1997, to be published in IEEE Trans. Nucl. Sci., June 1998.

4. Particle Data Group, "Review of particle properties", Physical Review D. Particles and Fields, Vol. 50, No. 3, p. 1338, 1994.

5. K. Gill et al. "Pion damage in semiconductor lasers," CMS Technical Note 1998. (available from the authors)

6. K. Gill et al. "Pion damage in p-i-n photodiodes," CMS Technical Note 1998. (available from the authors)

7. K. Gill et al., "Radiation damage studies of optoelectronics components for the CMS tracker optical links", Proceedings of Radiations and their Effects on Devices and Systems Conference (RADECS) 1997.

8. C. E. Barnes and J. J. Wiczer, "Radiation Effects in Optoelectronic Devices," Sandia Laboratory Report, SAND84-0771, 1984.

9. C. E. Barnes et al., "Effect of neutron irradiation on laser diode properties", SPIE Vol. 1174, pp. 233-261, 1989.

10. G. Shaw et al., "Radiation-induced reverse dark currents in InGaAs photodiodes," J. App. Phys. Vol. 73, pp. 7244-7249, 1993.

11. J. Troska et al., "Radiation damage studies of InGaAs p-i-n photodiodes for the CMS tracker optical links," CMS Technical Note 1997/102. (available from the authors).

12. H. Ohyama et al., "Degradation and recovery in $\operatorname{In}_{0.53} \mathrm{Ga}_{0.47} \mathrm{As}$ photodiodes by $1 \mathrm{MeV}$ neutrons," IEEE Trans. Nucl. Sci. Vol. 43 , No. 6, pp. 3019-3026, 1996.

13. P. W. Marshall et al., "Space-radiation effects on optoelectronic materials and components for a 1300nm fiber optic data bus," IEEE Trans. Nucl. Sci., Vol. 39, No. 6, pp. 1982-1989, 1992.

14. B. D. Evans et al., " $5.5 \mathrm{MeV}$ proton irradiation of a strained quantum-well laser diode and a multiple quantum-well broad-band LED,” IEEE Trans. Nucl. Sci., vol.40, pp. 1645-1654, 1993.

15. I. Dawson et al., "Irradiation tests of optoelectronic components for LHC inner-detectors," Nucl. Inst. and Meth. A 387, pp. 369-376, 1997.

16. RD20 Collaboration, "Radiation tolerance of single-sided silicon microstrips," Nucl. Inst. and Meth. A 339, pp. 511-523, 1994.

17. F. Vasey. CMS Tracker Optical Readout Link Specification. Available on the world-wide-web at:

http://www.cern.ch/CERN/Divisions/ECP/CME/OpticalLinks/\#Documents

18. Y. F. Zhao et al., "200MeV proton damage effects on multi-quantum well laser diodes", IEEE Trans. Nucl. Sci., Vol.44, No. 6, pp. 1898-1905, 1997.

19. A. Chilingarov et al., "Radiation damage due to NIEL in GaAs particle detectors", Nucl. Instr. and Meth. A.395 pp. 35-44, 1997.

20. S. J. Bates et al., "Pion-induced damage in silicon detectors," Nucl. Instr. and Meth. A.379 pp. 116-123, 1996.

21. E. A. Burke et al., "Energy dependence of proton-induced displacement damage in gallium arsenide", IEEE Trans. Nucl. Sci., Vol.34, No. 6, pp. 1220-1226, 1987.

22. J. Lindhard et al., "Integral equations governing radiation effects," Mat. Fys. Medd. K. Dan. Vidensk. Selsk. 33, pp. 1-41, 1963.

23. M. J. Norgett et al., "A proposed method of calculating displacement dose rates," Nucl. Eng. and Des., 33, pp. 50-54, 1975. 